\title{
FLOW BETWEEN COAXIAL ROTATING DISKS: WITH AND WITHOUT EXTERNALLY APPLIED MAGNETIC FIELD*
}

\author{
R.K. BHATNAGAR \\ Instituto de Matemática \\ Estatística e Ciência da Computação \\ Universidade Estadual de Campinas,13.100 \\ Campinas - São Paulo - Brasil
}

(Received May 28, 1979)

ABSTRACT. The problem of flow of a Rivlin-Ericksen type of viscoelastic fluid is discussed when such a fluid is confined between two infinite rotating coaxial disks. The governing system of a pair of non-linear ordinary differential equation is solved by treating Reynolds number to sma11. The three cases discussed are: (1) one disks is held at rest while other rotates with a constant angular velocity, (ii) one disk rotates faster than the other but in the same sense and (iii) the disks rotate in op posite senses and with different angular velocities. The radial, tranverse and axial components of the velocity field are plotted for the above three cases for different values of the Reynolds number. The results obtained for a viscoelastic fluid are compared with those for a Newtonian fluid. The velocity field for case (i) is also computed when a magnetic field is applied in a direction perpendicular to the discs and the results are compared with the case when magnetic field is absent. Some interesting features are observed for a viscoelastic fluid. 
KEY WORDS AND PHRASES. Viscoelastic fluid, rotating system, Newtonian fluid motion, perturbation solution.

1980 MATHEMATICS SUBJECT CLASSIFICATION CODES. 76A10, $76 A 05$.

\section{INTRODUCTION.}

In a recent investigation we have discussed the flow of a viscoelastic fluid of Rivlin-Ericksen type between a pair of infinite, coaxial rotating disks (see ref. $[1]$ and all the other references therein). The disks are taken to rotate with different constant angular velocities, either, in the same senses or opposite senses, or, one disk is held at rest and the other is taken to rotate with a constant angular velocity. The system of non-linear ordinary differential equations governing the flow was obtained and solved numerically under the appropriate boundary conditions of the problem using finite-difference technique and successive overrelaxation procedure. The solutions were given for values of the Reynolds number up to 1000 and some interesting features for the flow of a viscoelastic fluid were reported.

In the present investigation, therefore, it is our aim to discuss the problem of flow of a Rivlin-Ericksen fluid between coaxial rotating disks but for small values of the Reynolds number. As the basic system of equations governing such a flow has been already derived in [1], we shall briefly formulate the problem in the next section. In section 3, we obtain the pertubation solutions for small values of the Reynolds number. In section 4, we treat the flow in the presence of an externally applied magnetic field and provide pertubation solutions for the case of one disk at rest. At the end in section 5, we give some interesting illustrative examples for radial, transverse and axial components of the velocity and discuss characteristics of the flow field. 


\section{FORMULATION OF THE PROBLEM.}

Let us consider the steady flow of a Rivlin - Ericksen type of non-Newtonian fluid occupying the space between a pair of infinite parallel disks. In a cylindrical polar co-ordinate system $(r, \theta, z)$, let the lower disk situated at $z=0$ have an angular velocity $\Omega S$ while the other disk, situated at $z=d$ have an angular velocity $\Omega, \Omega$ and $\mathrm{S}$ being constants. Let $\mathrm{u}, \mathrm{v}$ and $\mathrm{w}$ represent the components of velocity in the increasing directions of $r, \theta$ and $z$ respectively.

We now write

$$
\mathrm{u}=-\frac{1}{2} \mathbf{r} \Omega \mathrm{H}^{\prime}(\eta), \mathrm{v}=\mathbf{r} \Omega \mathrm{G}(n), \quad \mathrm{w}=\mathrm{d} \Omega \mathrm{H}(n),
$$

so that the equation of continuity is identically satisfied for an axi-symmetric flow. Here $n$ is a non-dimensional axial co-ordinate given by $\eta=z / d$ and a dash denotes differentiation with respect to $\eta$.

Substituting from (1) into the equations of motion (as was done in [1] and eliminating the pressure from the equations in the radial and axial directions respective$1 y$, we obtain the following system of non-linear ordinary differential equations for. the functions $G$ and $H$ :

$$
\begin{aligned}
& G^{\prime \prime}+R\left(H^{\prime} G-H G^{\prime}\right)+\alpha R\left(H^{\prime} G^{\prime \prime}-H^{\prime} G^{\prime}\right) \\
& +\mathrm{KR}\left(\mathrm{HG}^{\prime \prime \prime}-\mathrm{H}^{\prime} \mathrm{G}^{\prime}\right)=0 \text {, } \\
& \mathrm{H}^{\mathrm{iv}}-\mathrm{R}\left(\mathrm{HH}^{\prime}{ }^{\prime}+4 \mathrm{GG}^{\prime}\right)+\alpha R\left(12 \mathrm{G}^{\prime} \mathrm{G}^{\prime}+\mathrm{H}^{\prime} \mathrm{H}^{\mathrm{iv}}+2 \mathrm{H}^{\prime} \mathrm{H}^{\prime} \mathrm{\prime}^{\prime}\right)+ \\
& +2 K R\left(H^{\prime} H^{\prime \prime \prime}+8 G^{\prime} G^{\prime}+\frac{1}{2} H^{\prime} H^{i v}+\frac{1}{2} H^{\vee}\right)=0 \text {, }
\end{aligned}
$$

where

$$
R=\frac{d^{2} \Omega \rho}{\phi_{1}} \text { is the Reynolds number and } \alpha=\frac{\phi_{3}}{\rho d^{2}}, K=\frac{\phi_{2}}{\rho d^{2}}
$$

are the non-dimensional parameters characterising the viscoelasticity of the fluid.

The equations (1) and (3) have to be solved under the following non-dimensional- 
ized boundary conditions:

$$
\begin{array}{lll}
G(0)=S & , & G(1)=1, \\
H(0)=0 & , & H(1)=0, \\
H^{\prime}(0)=0 & , & H^{\prime}(1)=0 .
\end{array}
$$

When $\alpha=0, K=0$, the above equations (2) and (3) reduce to the well-known equations to the corresponding problem for a classical viscous fluid.

\section{SOLUTION FOR SMALL VALUES OF REYNOLDS NUMBER $\mathbf{R}$.}

We now develop regular pertubation solutions for the functions $G$ and $H$ in the following manner:

$$
\begin{aligned}
& G(n)=G_{0}(n)+R G_{1}(n)+R^{2} G_{2}(n)+R^{3} G_{3}(n)+\ldots, \\
& H(n)=H_{0}(n)+R H_{1}(n)+R^{2} H_{2}(n)+R^{3} H_{3}(n)+\ldots .
\end{aligned}
$$

In order to obtain the various order solutions for the functions $G$ and $H$, we substitute their expressions in the equations (2) and (3) and the boundary conditions (4).

\section{(i) ZERO-ORDER SOLUTION}

The equations for $G_{0}$ and $H_{0}$ have the form

$$
G_{0}^{\prime \prime}=0
$$

and

$$
\mathrm{H}_{0}^{\mathrm{iv}}=0
$$

to be solved under the boundary conditions

$$
G_{0}(0)=S, G_{0}(1)=1, H_{0}(0)=H_{0}(1)=H_{0}^{\prime}(0)=H_{0}^{\prime}(1)=0 \text {. }
$$

It can be easily seen that 


$$
\begin{aligned}
& G_{0}(n)=s+(1-s) n, \\
& H_{0}(n)=0
\end{aligned}
$$

\section{(ii) FIRST-ORDER SOLUTION}

Making use of (9) and (10), the equations for $G_{1}$ and $H_{1}$ take the form

$$
G_{1}^{\prime \prime}=0
$$

and

$$
\mathrm{H}_{1}^{\mathrm{iv}}=4(1-\mathrm{S})[\mathrm{s}+(1-\mathrm{s}) \eta]
$$

The conditions satisfied by $\mathrm{G}_{1}$ and $\mathrm{H}_{1}$ are

$$
G_{1}(0)=G_{1}(1)=0, H_{1}(0)=H_{1}(1)=H_{1}^{\prime}(0)=H_{1}^{\prime}(1)=0 \text {. }
$$

Solving (11) and (12) and making use of (13), we get,

$$
G_{1}(n)=0
$$

and

$$
\begin{aligned}
& \mathrm{H}_{1}(\eta)=\frac{1}{30}(1-\mathrm{S})(2+3 \mathrm{~S}) \eta^{2}-\frac{1}{30}(1-\mathrm{S})(3+7 \mathrm{~S}) \eta^{3} \\
& +\frac{1}{6} \mathrm{~s}(1-\mathrm{S}) \eta^{4}+\frac{1}{30}(1-\mathrm{s})^{2} \eta^{5} .
\end{aligned}
$$

It may be noted that the zero and first order solutions do not involve the viscoelastic parameters $\alpha$ and $K$.

\section{(iii) SECOND-ORDER SOLUTION}

It can be easily seen that the equations determining the functions $G_{2}$ and $\mathrm{H}_{2}$ have respectively the forms

$$
G_{2}^{\prime \prime}=\left(H_{1} G_{0}^{\prime}-H_{1}^{\prime} G_{0}\right)+(\alpha+K) H_{1}^{\prime} G_{0}^{\prime},
$$

and 


$$
\mathrm{H}_{2}^{\mathrm{iv}}=0
$$

to be solved under the boundary conditions

$$
G_{2}(0)=G_{2}(1)=0 ; H_{2}(0)=H_{2}(1)=H_{2}^{\prime}(0)=H_{2}^{\prime}(1)=0 \text {. }
$$

Thus we get,

$$
\begin{aligned}
& G_{2}(\eta)=\frac{1-s}{6300}\left(27 s^{2}+16 s-8\right) \eta-\frac{s(1-s)}{90}(2+s s) n^{3} \\
& -\frac{1-s}{180}\left(1-4 s-12 s^{2}\right) \eta^{4}+\frac{1-s}{300}\left(3+4 s-17 s^{3}\right) n^{5} \\
& -\frac{1}{45} s(1-s)^{2} \eta^{6}-\frac{1}{315}(1-s)^{3} n^{7} \\
& +(\alpha+K)\left[\frac{(1-s)^{2}}{30}(2+3 s) n^{2}-\frac{(1-s)^{2}}{30}(3+7 s) n^{3}\right. \\
& \left.+\frac{1}{6} s(1-s)^{2} n^{4}+\frac{1}{30}(1-s)^{3} n^{5}\right],
\end{aligned}
$$

and

$$
\mathrm{H}_{2}(n)=0 \text {. }
$$

\section{(iv) THIRD-ORDER SOLUTION}

Substituting from (5) into the equations (2) and (3), it may be easily verified that $G_{3}$ and $H_{3}$ satisfy the following equations respectively:

$$
\begin{aligned}
& G_{3}^{\prime \prime}=0, \\
& H_{3}^{i v}-\left(H_{1} H_{1}^{\prime \prime \prime}+4 G_{0} G_{2}^{\prime}+4 G_{0}^{\prime} G_{2}\right) \\
& +\alpha\left(12 G_{0}^{\prime} G_{2}^{\prime \prime}+12 G_{0}^{\prime \prime} G_{2}^{\prime}+H_{1}^{\prime} H_{1}^{i v}+2 H_{1}^{\prime} H_{1}^{\prime \prime}\right) \\
& +2 K\left(8 G_{0}^{\prime} G_{2}^{\prime \prime}+8 G_{0}^{\prime} G_{2}^{\prime}+H_{1}^{\prime} H_{1}^{\prime \prime}+\frac{1}{2} H_{1}^{\prime} H_{1}^{i v}+\frac{1}{2} H_{1} H_{1}^{v}\right)=0 .
\end{aligned}
$$


Using the expressions for $G_{0}, G_{2}$ and $H_{1}$ in the above equations and solving them under the prescribed boundary conditions on $G_{3}, H_{3}$ and $H_{3}^{\prime}$ we find that, the third-order solution is given by,

$$
\begin{aligned}
G_{3}(n) & =0, \\
H_{3}(n) & =x_{0} n^{2}+x_{1} n^{3}+x_{2} n^{4}+x_{3} n^{5} \\
& +x_{4} n^{6}+x_{5} n^{7}+x_{6} n^{8}+x_{7} n^{9} \\
& +x_{8} n^{10}+x_{9} n^{11},
\end{aligned}
$$

where $x_{i}, i=0$ to $i=9$ are given by

$$
\begin{aligned}
& x_{0}=-\left[\frac{S(1-s)}{226800}\left(461-922 s-1219 s^{2}\right)+\frac{1-s}{2268000}(332\right. \\
& \left.\left.-1011 s+2466 s^{2}+913 s^{3}\right)\right]-\frac{\alpha(1-s)^{2}}{567000}(474-1713 s \\
& \left.-2361 s^{2}\right)-\frac{K(1-s)^{2}}{283500}\left(552-1059 s-1743 s^{2}\right) \\
& +\frac{1}{3150}(\alpha+K)(3 \alpha+4 K)(1-S)^{3}(16+19 S) \text {, } \\
& x_{1}=\frac{S(1-S)}{226800}\left(856-1712 s-2504 s^{2}\right)+\frac{1-s}{2268000}(579-1277 s \\
& \left.+3117 s^{2}+1781 s^{3}\right)-\frac{\alpha(1-s)^{2}}{567000}\left(839+8072 s+8189 s^{2}\right) \\
& -\frac{K(1-s)^{2}}{283500}\left(7+4636 s+5257 s^{2}\right)+\frac{1}{3150}(\alpha+K)(3 \alpha+4 K)(1-s)^{4} \text {, } \\
& x_{2}=-\frac{1}{4725} s(1-s)\left(8-16 s-27 s^{2}\right)+\frac{(\alpha+K)}{900}(1-s)^{2}(2+3 s)(3+7 s) \\
& -\frac{1}{90}(\alpha+K)(3 \alpha+4 K)(1-S)^{3}(2+3 S),
\end{aligned}
$$




$$
\begin{aligned}
& x_{3}=-\frac{1}{94500}(1-s)^{2}\left(8-16 s-27 s^{2}\right)-\frac{1}{4500}(1-s)^{2}[\alpha(27+106 s \\
& \left.\left.+117 s^{2}\right)+K\left(27+86 s+87 s^{2}\right)\right]+\frac{(\alpha+K)(3 \alpha+4 K)}{150}(1-s)^{3}(3+7 s) \text {, } \\
& x_{4}=-\frac{1}{54000}(1-s)(2+3 s)\left(3+4 S+13 s^{2}\right)+\frac{(1-s)^{2}}{2700}[\alpha(4+29 s \\
& \left.\left.+57 s^{2}\right)+k\left(4+20 s+36 s^{2}\right)\right]-\frac{1}{45}(\alpha+k)(3 \alpha+4 K) s(1-s)^{3} \text {, } \\
& x_{5}=\frac{1}{378000}(1-S)\left(27+19 s+161 s^{2}+393 s^{3}\right) \\
& +\frac{(1-s)^{2}}{315}\left[\frac{\alpha}{2 \sigma}\left(3+4 s^{-}-67 s^{2}\right)-2 K s^{2}-(\alpha+k)(3 \alpha+4 K)(1-s)^{2}\right], \\
& x_{6}=\frac{1}{151200}(1-s)\left(2+17 s+20 s^{2}-99 s^{3}\right)-\frac{\alpha}{36 \theta} s(1-s)^{3}-\frac{K}{630} s(1-s)^{3}, \\
& x_{7}=\frac{1}{453600}(1-s)^{2}\left(3+4 s-107 s^{2}\right)-\frac{(1-s)^{4}}{22680}(7 \alpha+4 K), \\
& x_{8}=-\frac{11}{226800} s(1-s)^{3}, \\
& x_{9}=-\frac{1}{226800}(1-s)^{4} .
\end{aligned}
$$

Thus the components of the velocity field $u, v$ and $w$ have been computed to the third power of the Reynolds number $R$.

4. EFFECTS OF TRANSVERSE MAGNETIC FIELD ON THE FLOW FIELD :CASE OF ONE DISK HELD AT REST.

We now consider the problem of flow of a viscoelastic fluid when a constant magnetic field $\overline{\mathrm{H}}_{0}$ is applied in a direction perpendicular to the discs. Making use of the steady state Maxwell's equations and adding the contributions due to Lorentz force in the equations of motion, it can be verified that the equations (2) and 
for the functions $G$ and $H$ are modified to have the following forms respectively:

$$
\begin{aligned}
G^{\prime \prime} & +R\left(H^{\prime} G-H G^{\prime}\right)+\alpha R\left(H^{\prime} G^{\prime} '-H^{\prime} G^{\prime}\right) \\
& +K R\left(H G^{\prime}+'-H^{\prime} G^{\prime}\right)-M^{2} G=0,
\end{aligned}
$$

and

$$
\begin{aligned}
& \mathrm{H}^{\mathrm{iv}}-\mathrm{R}\left(\mathrm{HH}^{\prime}{ }^{\prime}+4 \mathrm{GG}^{\prime}\right)+\alpha R\left(12 \mathrm{G}^{\prime} \mathrm{G}^{\prime}+\mathrm{H}^{\prime} \mathrm{H}^{\mathrm{iv}}+2 \mathrm{H}^{\prime} \mathrm{H}^{\prime}{ }^{\prime}\right) \\
& +2 \mathrm{KR}\left(8 \mathrm{G}^{\prime} \mathrm{G}^{\prime \prime}+\mathrm{H}^{\prime} \mathrm{H}^{\prime}{ }^{\prime}+\frac{1}{2} \mathrm{H}^{\prime} \mathrm{H}^{\mathrm{iv}}+\frac{1}{2} \mathrm{HH}^{\mathrm{V}}\right)-\mathrm{M}^{2} \mathrm{H}^{\prime}{ }^{\prime}=0,
\end{aligned}
$$

where the Hartmann number $M$ is defined as

$$
M^{2}=\frac{\mu_{0}^{2} \overline{\mathrm{H}}_{0}^{2} \mathrm{~d}^{2} \sigma}{\rho \phi_{1}} .
$$

In the above expression for $M, \mu_{0}$ represents the magnetic permeability and $\sigma$ the conductivity.

The boundary conditions satisfied by $G(n)$ and $H(n)$ in equations (35) and (36) are the same as in (4).

We shall now obtain solutions for the case of one disk at rest, namely, the case with $S=0$. Once again treating Reynolds number $R$ to be samall, we expand the functions $G(\eta)$ and $H(n)$ in a series in $R$ as was done in (5). The zeroth, first and the second order solutions are then given as follows:

\section{(i) ZERO-ORDER SOLUTION}

When $M \neq 0$, the equations for $G_{0}$ and $H_{0}$ are

$$
\begin{aligned}
& G_{0}^{\prime \prime}-M^{2} G_{0}=0 \\
& H_{0}^{i v}-M_{0}^{2} H^{\prime \prime}=0,
\end{aligned}
$$

Solving (37) and (38) and using the boundary conditions (8), it can be easily seen that

$$
G_{0}(n)=\frac{1}{\operatorname{Sinh} M} \sinh (M n),
$$


and

$$
\mathrm{H}_{0}(n)=0 \quad \text {. }
$$

(ii) FIRST-ORDER SOLUTION

The functions $G_{1}(n)$ and $H_{1}(n)$ satisfy the following equations:

$$
\begin{gathered}
G_{1}^{\prime \prime}-M^{2} G_{1}=0, \\
H_{1}^{i v}-M^{2} H_{1}^{\prime \prime}=4 G_{0} G_{0}^{\prime}-4(3 \alpha+4 K) G_{0}^{\prime} G_{0}^{\prime \prime} .
\end{gathered}
$$

Solving them under the boundary conditions (13), we get

$$
\begin{gathered}
G_{1}(n)=0 \\
H_{1}(n)=C+D n+\frac{A}{M^{2}} \operatorname{Cosh}(M n)+\frac{B}{M^{2}} \sinh (M n)+a_{0} \sinh (2 M n),
\end{gathered}
$$

where

$$
a_{0}=\frac{1}{6 M^{3} \sinh ^{2} M}\left[1-M^{2}(3 \alpha+4 K)\right],
$$

$$
\begin{aligned}
A= & \frac{a_{0}}{b_{0}} M^{2}[(1-\operatorname{Cosh} M) \sinh (2 M)+2(M \operatorname{Cosh} M-\sinh M) \\
& \quad+2(\sinh (M)-M) \operatorname{Cosh}(2 M)] . \\
B= & \frac{a_{0}}{b_{0}} M^{2}[2(1-\operatorname{Cosh} 2 M)(\operatorname{Cosh} M-1)-\sinh M(2 M-\sinh 2 M)], \\
C= & \frac{a_{0}}{b_{0}}[(\operatorname{Cosh} M-1) \sinh 2 M-2(M \operatorname{Cosh} M-\operatorname{Sinh} M) \\
D= & \frac{a_{0}}{b_{0}} M[-\sinh (M) \sinh (2 M)+2(\operatorname{Cosh} M-1)(1+\operatorname{Cosh} 2 M)],
\end{aligned}
$$


and

$$
\mathrm{b}_{0}=M \sinh (M)+2\left(1^{-}-\operatorname{Cosh} M\right)
$$

\section{(iii) SECOND-ORDER SOLUTION}

The equation satisfied by $G_{2}(n)$ is

$$
G_{2}^{\prime \prime}-M^{2} G_{2}=\left(1-M^{2} K\right) H_{1} \dot{G}^{\prime}-\left(1+M^{2} \alpha\right) H_{1}^{\prime} G_{0}+(\alpha+K) H_{1}^{\prime} G_{0}^{\prime} \text {. }
$$

Solving (51) under the appropriate boundary conditions, we have

$$
\begin{aligned}
& \mathrm{G}_{2}(\eta)=\mathrm{P}_{1}\{\operatorname{Cosh}(\mathrm{M} n)-1\}+\mathrm{P}_{2} \sinh (\mathrm{M} n)+\mathrm{P}_{3} \eta \operatorname{Cosh}(\mathrm{Mn}) \\
& +\mathrm{P}_{4}\left(2 \mathrm{Cn}+\mathrm{Dn}^{2}\right) \sinh (M n)+\mathrm{P}_{5} \sinh (3 M n),
\end{aligned}
$$

where $P_{1}, P_{2}, P_{3}, P_{4}$ and $P_{5}$ are given by

$$
\begin{aligned}
P_{1} & =\frac{A}{M^{3} \sinh (M)}\left(1+M^{2}(x)\right. \\
P_{2} & =\frac{P_{1}}{\sinh M}(1-\cosh M)-\frac{\cosh M}{\sinh ^{2}(M)} \mid \frac{3 a}{4}\left\{1+y^{2}(2+h)\right. \\
& -\frac{D}{4 M}\left(3-M^{2} K\right)-\frac{M D \alpha}{2} \mid-\frac{2 C+D}{4 \sinh M}\left(1-M^{2} K^{\prime}\right) \\
& \left.+\frac{a_{0} \sinh (3 M)}{16 M \sinh (M)}{ }^{2}-M^{2}(3 K+2 \alpha)\right\}, \\
P_{3} & =\frac{1}{\sinh (M)}\left[\frac{3 a}{4}\left\{1+M^{2}(2 \alpha+K)\right\}-\frac{D}{4 M}\left(3-M^{2} K\right)-\frac{M D \alpha}{2}\right] \\
P_{4} & =\frac{1}{4 \operatorname{Sinh}(M)}\left(1-M^{2} K\right)
\end{aligned}
$$

and

$$
P_{5}=-\frac{a_{0}}{16 M \sinh (M)}\left\{1-M^{2}(3 K+2 \alpha)\right\} \text {. }
$$


As for the case with $M=0$, here also it can be verified that

$$
M_{2}(n)=0
$$

This completes the solution for the case of one disk at rest in the presence of tranverse magnetic field. The solutions for the case $S \neq 0$ will be presented separately.

\section{DISCUSSION OF THE RESULTS}

(i) Lower disk held at rest or rotating faster than the upper disk:

Throughout our discussion we have taken the values of the Reynolds number $R$ to be $R=0.2,0.4,0.6$ and 0.8 . For the sake of comparison, results are depicted for a classical viscous fluid $(\alpha=0, K=0)$ and a typical viscoelastic fluid for which $\alpha=0.1$ and $K=-0.05$.

Fig. 1 depicts the curves of $H$ for a classical viscous fluid $\alpha=0, K=0$ for $S=0$ and 5.0. For the case $S=0$, comparison is also made between the curves of $H$ when the Hartmann number $M=0$ and 1.0. It is noted that when $S=0$, the function $H$ representing the axial velocity is always positive whether $M=0$ or 1.0 . The effect of the tranverse magnetic field on the flow is to decrease the axial velocity for each value of the Reynolds number from $R=0.2$ to $R=0.8$. The profiles are nearly parabolic in character. When $S=1$, the whole system undergoes a rigid body notation and as $S$ increases further, the function $M$ changes its sign. This may be observed from the curves depicting axial velocity profiles for $S=5.0$ and $R=0.2$, $0.4,0.6$ and 0.8 in Fig. 1 .

In Fig. 2, we have drawn the axial velocity profiles for a viscoelastic fluid $\alpha=0.1, K=-0.05$ for $S=0$ and 5.0. The values of $R$ are same as in Fig. 1 . The cháfacter of the profiles is similer to those for a classical Newtonian fluid. It is interesting to note that in the presence of viscoelasticity in the fluid, the effect of tranverse magnetic field is to decrease the axial velocity much more than for a Newtonian liquid. This may be easily observed from the curves of $\mathrm{H}$ for $M=1.0$ 
and comparing them with the corresponding curves in Fig. 1 for $\alpha=0, \mathrm{~K}=0$. Here also for $S=5.0$, i.e. when the lower disk rotates five times faster the upper disk, the axial velocity becomes completely negative.

Fig. 3 represents the curves for the function $H^{\prime}$ for $S=0$ and $\alpha=0.1, K=-0.05$. It may be recalled that the radial velocity is actually designated by $-\mathrm{H}^{\prime}$. For each value of the Reynolds number $R$ from $R=0.2$ to $0.8, H^{\prime}$ is positive in the lower half region whereas it is negative in the upper half region between the two disks. The increase in $\mathrm{R}$ gives rise to increase in $\mathrm{H}^{\prime}$ in the lower half region. The reverse is true in the upper half region. Once again, it is observed that the presence of tranverse magnetic field decreases $H^{\prime}$ for each $R$ in the lower half region and that the reverse holds for the upper half region.

In Fig. 4. we have drawn the profiles of the function $\mathrm{H}^{\prime}$ for $\alpha=0.1, \mathrm{~K}=-0.05$ and $S=5.0$. Comparing these curves with the corresponding curves for $S=0$ in $\mathrm{Fig} \cdot 4$, we note that the flow field is reversed and now $\mathrm{H}^{\prime}$ is negative in the lower half region whereas it is positive in the upper.half region between the two disks.

Fig, 5 represents the curves of the tranverse velocity function $G$ for the case of lower disk held at rest i.e. $S=0$, the fluid parameters being $\alpha=0.1, K=-0.05$. Choosing the value of the Reynolds number $R=0.2$, comparison is made for Hartmann number $M=0$ and 1 . In the absence of magnetic field, $G$ varies linearly from $G=0$ at the lower disk to $G=1$ at the upper disk. The tranverse velocity is increased when magnetic field is applied as it is clear from the curve of $G$ for $M=1.0$. The situation is contrary in Fig. 6, which shows curves of $G$ for $R=0.4$ and $\alpha=0.1, K=-0.05$, the lower disk rotating five times faster than the upper disk, G now decreases 1 inear$1 y$ between the two disks from $G=5$ at the lower disk to $G=1$ at the upper disk.

(ii) Two disks rotating in opposite directions and with different angular velocities:

We now discuss some results for a viscoelastic fluid $\alpha=1, \mathrm{~K}=-0.05$ when the 
two disks rotate in opposite directions and with different angular velocities i.e. $S$ is non-zero negative.

Fig. 7 depicts the curves of the axial velocity function $H$ for $R=0.2,0.4$, $0.6,0.8$ and different negative values of $\mathrm{S}$. When $\mathrm{S}=-0.5, \mathrm{H}$ is completely positive and increases in the entire region with increase in $R$. However, when $S=-1.0$, i.e. when the two disks rotate with same angular velocity but in opposite senses, $\mathrm{H}$ is positive but only in the upper half region. In the lower half region, it takes negative values. They are not shown in the figure because of their extreme smallness. For $\mathrm{S}=-0.5$, the region of positive $\mathrm{H}$ completely disappears and the axial velocity becomes negative in the entire region between the two disks. In this case, therefore $H$ decreases with increase in $R$.

In Fig. 8, we have drawn the curves of $H^{\prime}$ for $S=-0.5$ and -1.0 . When $S=-0.5$, $\mathrm{H}^{\prime}$ takes both positive and negative values. The point where $\mathrm{H}^{\prime}$ vanishes lies in the upper half region $(n \simeq 0.57)$. The increase in $R$ increases $H^{\prime}$ in the lower region and reverse is true for the upper region. The situation no longer remains so when $S=-1.0$. The flow region is now divided into three parts with the appearance of a central core in which $H^{\prime}$ is positive for all values of $\mathrm{R}$ considered. In the other two regions, one near the lower disk and the other near the upper disk, $\mathrm{H}^{\prime}$ is negative. Thus for this case, we have two points where $\mathrm{H}^{\prime}$ vanishes; one lies in the lower half plane whereas the other lies in the upper half plane.

From Fig. 9 showing the curves of $\mathrm{H}^{\prime}$ for $\mathrm{S}=-0.5$, we observe that the central core has disappeared. Now $\mathrm{H}^{\prime}$ is negative in the lower region whereas it is positive in the upper region. The point where $\mathrm{H}^{\prime}$ vanishes lies in the lower half plane. The increase in $\mathrm{R}$ causes increase in $\mathrm{H}^{\prime}$ in the upper half region while the reverse is true for the lower half plane between the two disks.

Fig. 10 represents the graph for the function $G$ depicting the tranverse velocity for $\mathrm{R}=0.6, \mathrm{~S}=-5.0$ and $\alpha=0.1, \mathrm{~K}=-0.05$. It is observed that $\mathrm{G}$ varies linearly from -5.0 at $n=0$ to 1.0 at $n=1.0$. This behaviour is similar to those for $S=0$ as $S=5.0$ in the absence of magnetic field.

The numerical computations were carried out on the University of Campinas PDP 10 computer. 

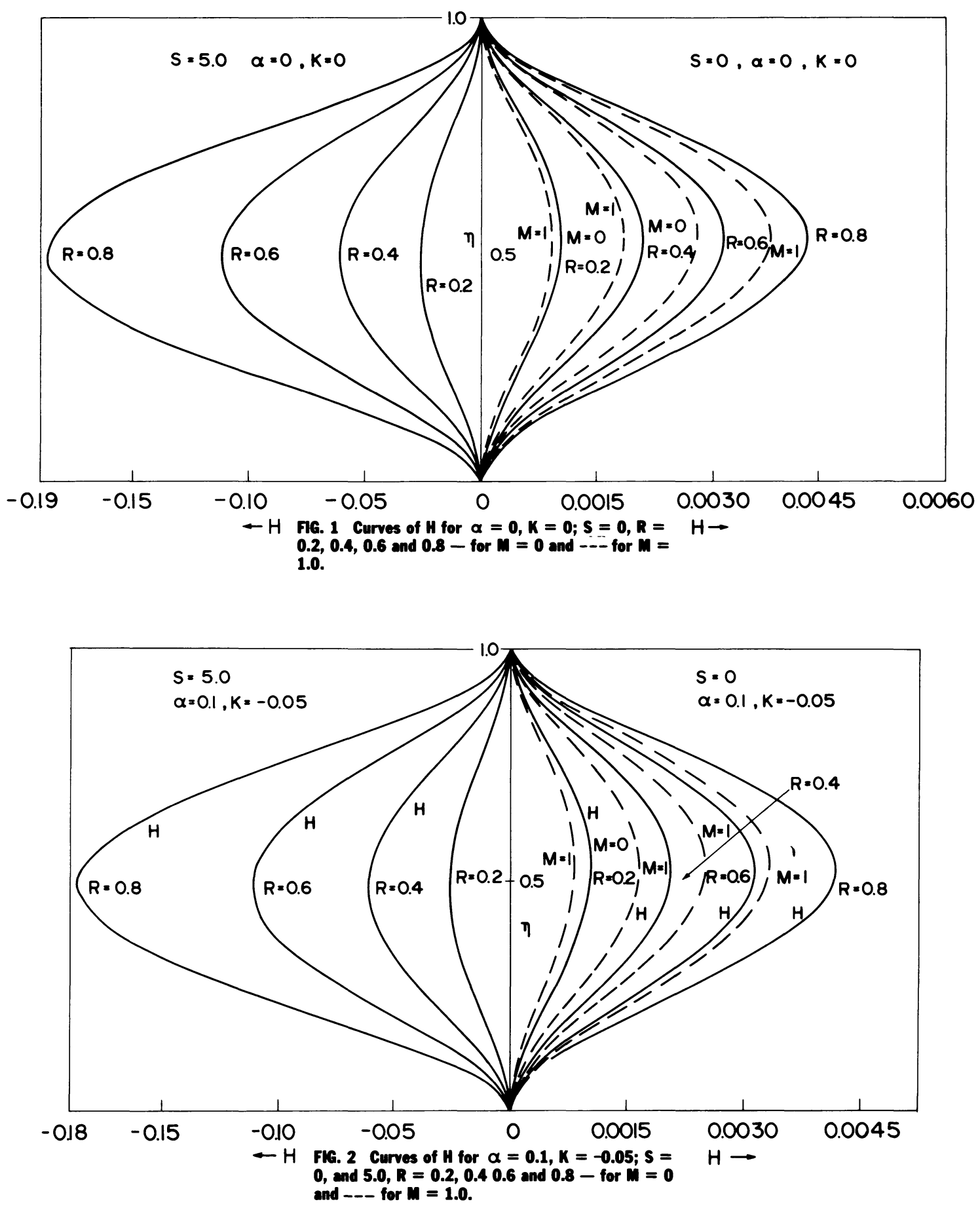

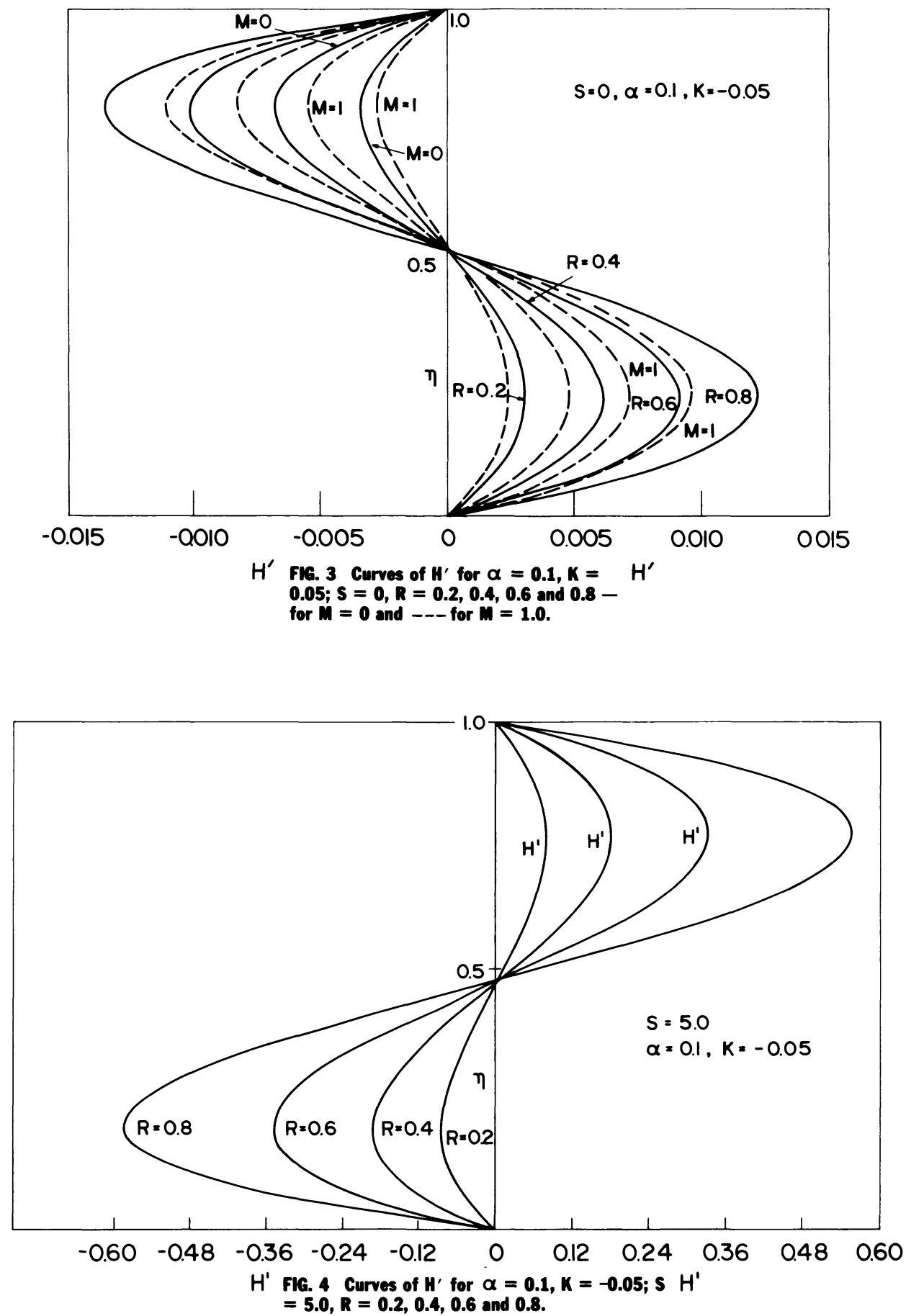
FLOW BETWEEN COAXIAL ROTATING DISKS

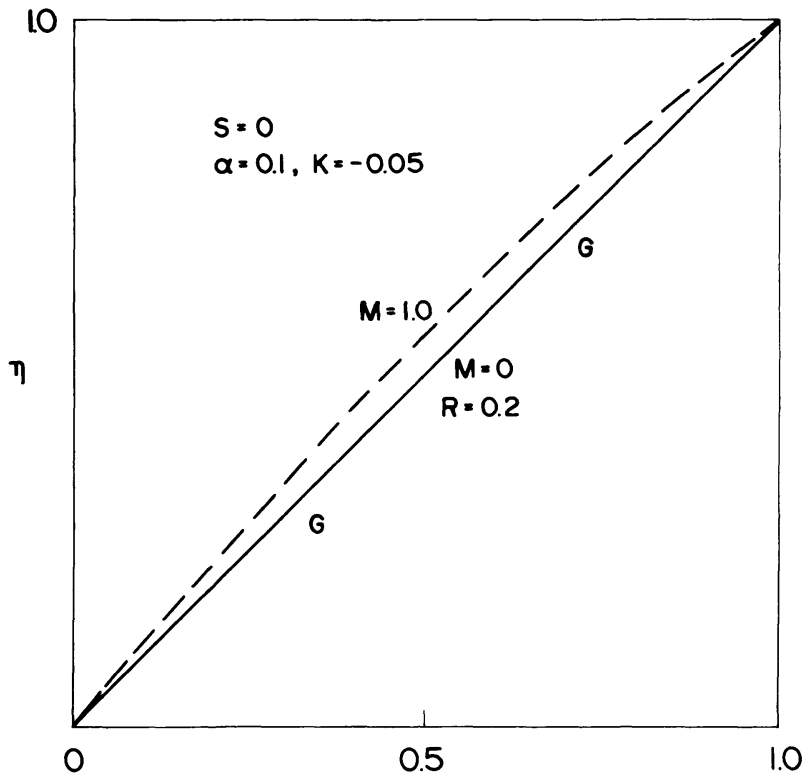

FIG. 5 Curves of $G$ for $\alpha=0.1, K=-0.05 ; S=0$ and $R=0.2$. - for $m=0$ and --- for $m=1.0$.

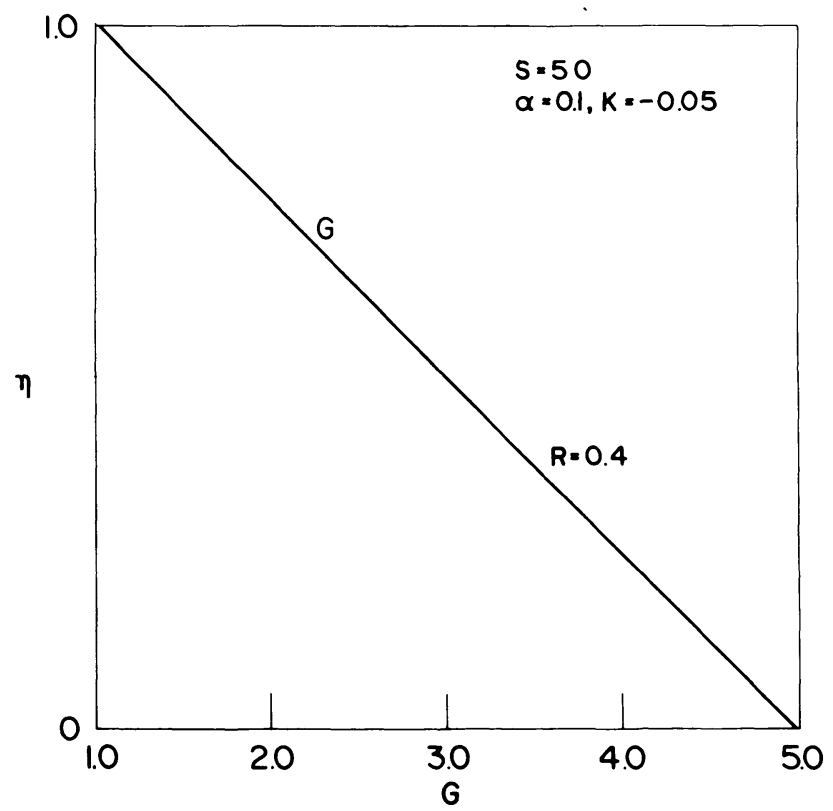

FIG. 6 Curves of $G$ for $\alpha=0.1, K=-0.05, S=0.5$ and $R=0.4$. 

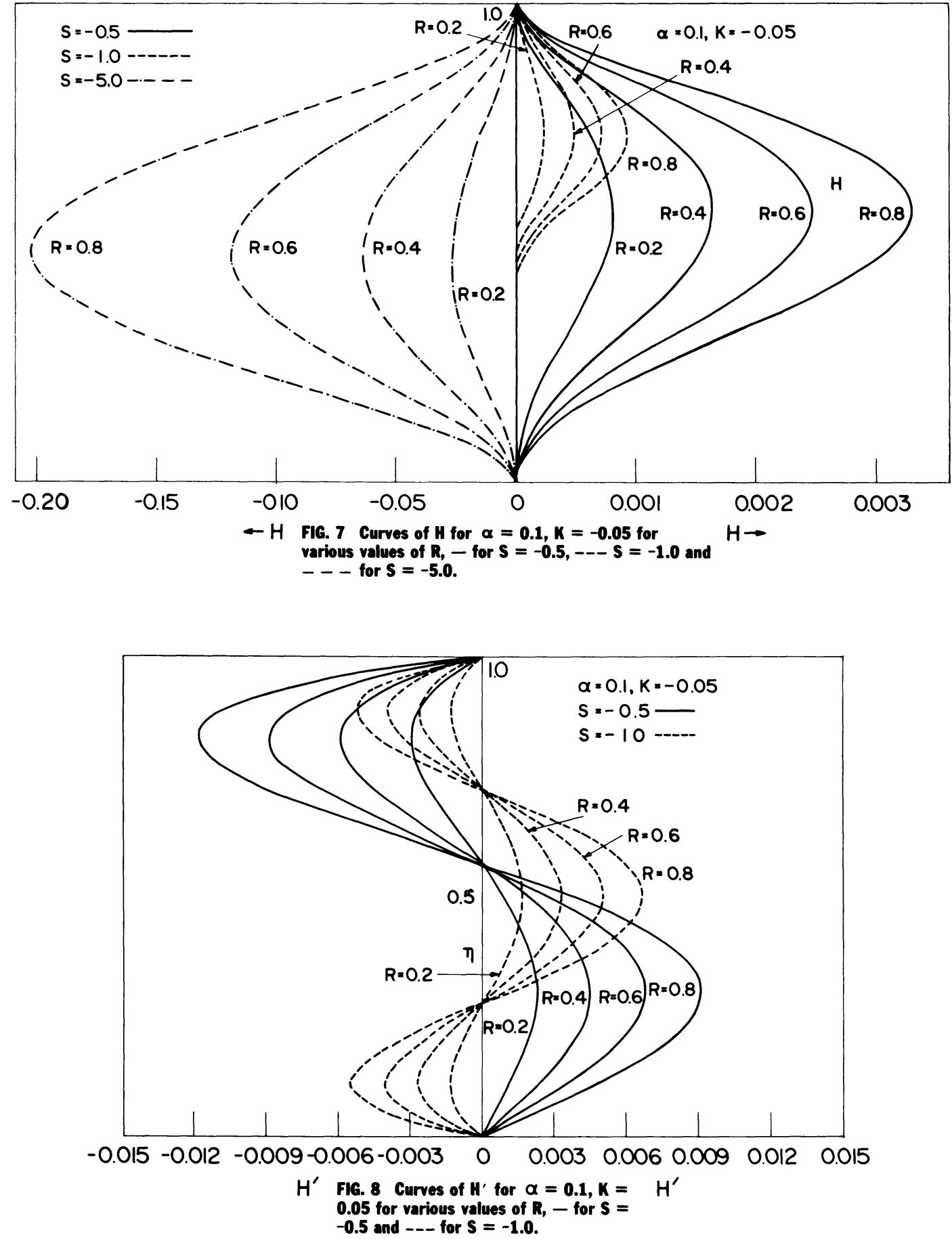

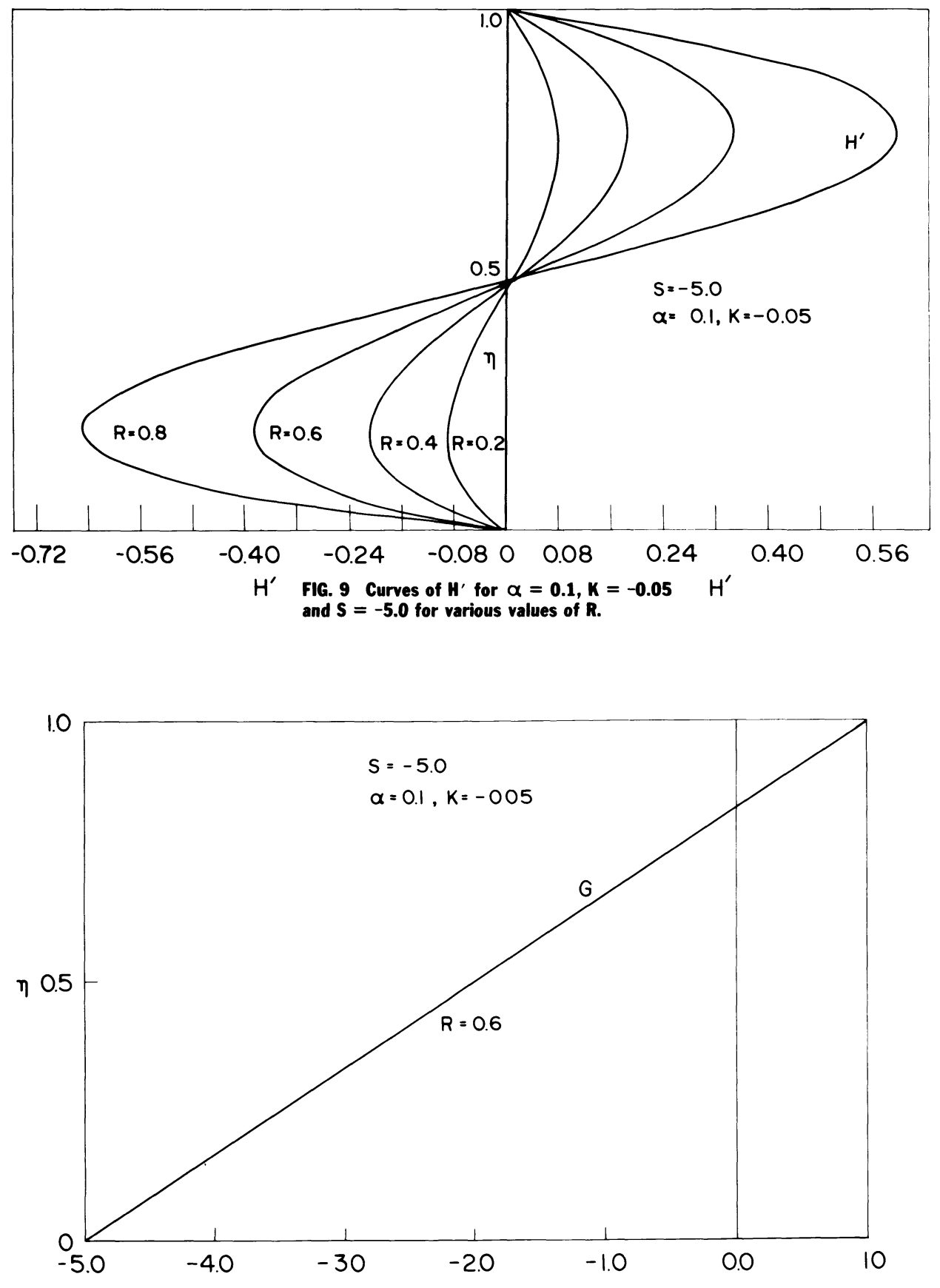

FIG. 10 Curve of $G$ for $\alpha=0.1, K=-0.05, S=-5.0$ and $R=0.6$. 
*The paper was presented at the IUTAM Symposium on Non-Newtonian fluid Mechanics

held at Louvain-la-Neuve, Belgium from Aug. 28 to Sept. 1, 1978.

\section{REFERENCES}

[1] Bhatnagar, R.K. and J.V. Zago. Numerical Investigations of Flow of a Viscoelastic fluid between Rotating Coaxial Disks, Rheologica Acta 17, (1978), 557-567. 


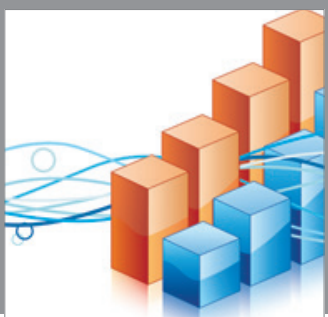

Advances in

Operations Research

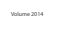

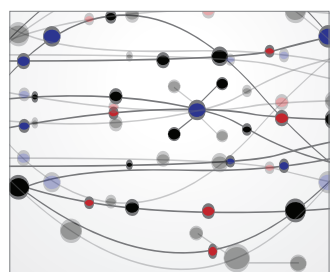

\section{The Scientific} World Journal
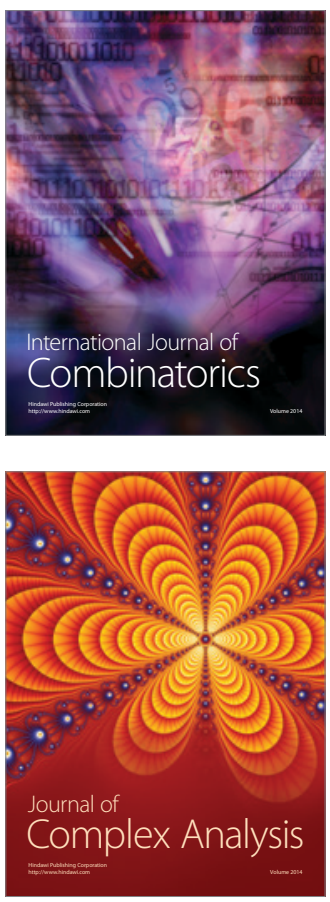

International Journal of

Mathematics and

Mathematical

Sciences
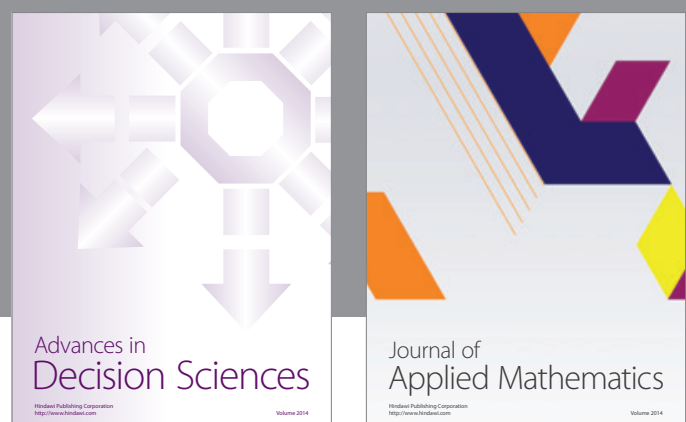

Journal of

Applied Mathematics
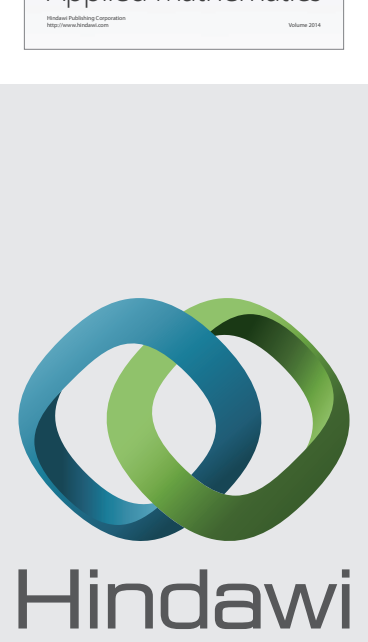

Submit your manuscripts at http://www.hindawi.com
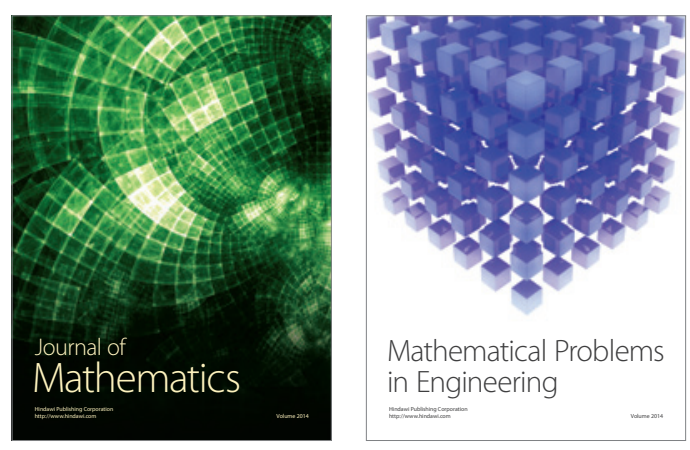

Mathematical Problems in Engineering
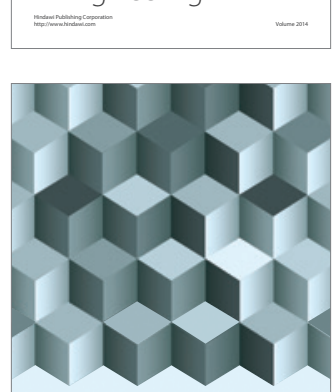

Journal of

Function Spaces
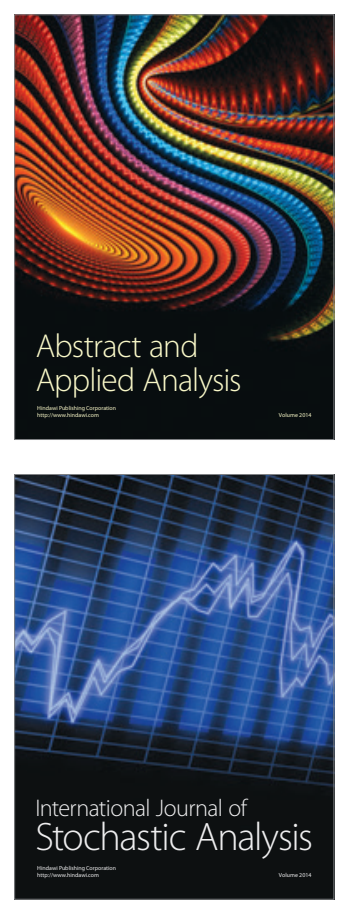

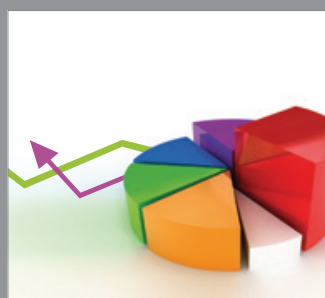

ournal of

Probability and Statistics

Promensencen
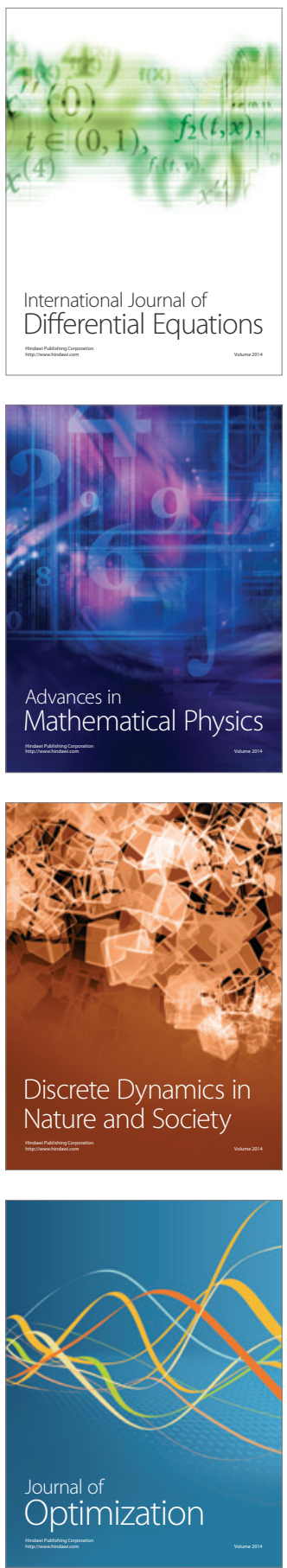